\title{
High Impact Practices-Encouraging empowerment and self-care through participatory women's groups and group antenatal care: Supporting women to be at the center of their own care as active participants
}

Tracy McClair

Population Council

Follow this and additional works at: https://knowledgecommons.popcouncil.org/departments_sbsr-rh

Part of the Maternal and Child Health Commons

How does access to this work benefit you? Let us know!

\section{Recommended Citation}

McClair, Tracy. 2020. "High Impact Practices-Encouraging empowerment and self-care through participatory women's groups and group antenatal care: Supporting women to be at the center of their own care as active participants," Hypertensive Disorders in Pregnancy brief. Washington, DC: Population Council. 


\section{HIGH IMPACT PRACTICES}

Encouraging empowerment and self-care through

participatory women's groups and group antenatal care

\section{Supporting women to be at the center of their own care as active participants}

\section{WHAT IS THE HIGH-IMPACT PRACTICE IN MATERNAL HEALTH?}

Encouraging health awareness, self-care and informed decision making through existing women's groups platforms can improve health seeking behaviors and maternal health service uptake. Highly participatory group-based interventions for women during the antenatal and postnatal periods provide information, education, and counseling for improved health literacy (including on the hypertensive disorders of pregnancy), social support, and opportunities to cultivate empowerment and agency. This brief will highlight the role of women's groups and group ANC in education and screening of pre-eclampsia.

\section{BACKGROUND}

Women's groups focusing on maternal and newborn health $(\mathrm{MNH})$ have been implemented in low and middle-income countries (LMICs) to bring care closer to women in their communities, support women's empowerment around their healthcare, and contribute to improved healthcare outcomes for women and their newborns. These groups occur in the antenatal and postnatal periods and can vary in terms of number of sessions, frequency of sessions, number of women in each session, whether or not the same group of women attend each session, and whether or not a clinical component is incorporated, among other variations. These groups offer opportunities to provide information about and screening for symptoms of pre-eclampsia and eclampsia, the second most common cause of pregnancyand child-related deaths among women globally.

\section{Participatory women's groups and group ANC as self-care interventions}

The White Ribbon Alliance promotes self-care interventions for improved maternal and child health because they

\section{KEY POINTS}

- Highly participatory, group-based interventions such as community-based women's groups and group antenatal and postnatal care (ANC/PNC) provide opportunities for women to share their experiences and knowledge with others, discuss the local context for challenges and how to address them, and promote empowerment and agency.

- Participation in these groups is associated with improved maternal health literacy, improved health behaviors, increased maternal health service uptake, and reduced maternal and neonatal mortality.

- Women's groups and group ANC are platforms for education and screening of pre-eclampsia.

are the root of healthcare, starting with women and their communities [4, 5]. The 2019 Word Health Organization's (WHO) "Consolidated Guideline on Self-Care Interventions for Health" defines self-care as, "the ability of individuals, families and communities to promote health, prevent disease, maintain health, and to cope with illness and disability with or without the support of a health-care provider" [6]. Self-care interventions - such as women's groups and group ANC - have the ability to promote choices and support informed decision making on health when a supportive enabling environment exists, and can be cost-effective, improve quality of care, and contribute to sustainable community health [6].

\section{WHO guidelines on women's groups}

WHO guidelines recommend women's groups for $\mathrm{MNH}$ particularly in rural areas with low access to health services [1]. The guidelines suggest that the groups can support women to generate and advocate for local solutions for $\mathrm{MNH}$.

The Population Council conducts research and delivers solutions that improve lives around the world. Big ideas supported by evidence:

It's our model for global change. popcouncil.org

(C) 2020 The Population Council, Inc.

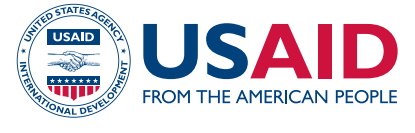
ENDING 


\section{WHO guidelines on group ANC}

Group ANC can be substituted for individual ANC in the context of rigorous research [7].

\section{Participation in women's groups has been associ-} ated with:

- Improved knowledge on: contraception, sexually transmitted infection (STI) treatment and prevention, nutrition, anemia prevention [8], hypertension and pregnancy complications, and the importance of ANC attendance [9]

- Collective efficacy [10]

- Positive healthcare behaviors including taking iron and folic acid [11], increased ANC uptake [11, 12], increased institutional deliveries [10, 12-14], skinto-skin care, exclusive breastfeeding, immunization, and increased contraceptive use [10]

- Reduced maternal and neonatal morbidity and mortality [15-18]

\section{Previous research has shown that group ANC is} associated with:

- Facility-based delivery and attending at least four ANC visits [19]

- Contraceptive use, exclusive breastfeeding for more than 6 months, improved health literacy, higher pregnancy-related empowerment, taking prenatal vitamins and iron, and higher satisfaction with services [20-22]

- Increased and more accurate information about pregnancy and delivery, social support, and women's empowerment from clients' perspectives [23]

- More comprehensive ANC services and improved experiences of care from providers' perspectives [24]; however, providers also express concerns about organizational and management challenges of implementing group ANC/PNC including additional staff time and space [23]

- Higher quality of care [19]

In sum, participatory women's groups and group ANC encourage women gathering for shared needs and interests in order to disseminate information, share
Definitions: Participatory women's groups and group ANC/PNC

- Participatory women's groups are community -based organized groups that support the mobilization of women and their communities to improve health or social outcomes, including maternal and newborn health outcomes. These women's groups operate within the community and address contextspecific barriers to health behavior change. Groups may be initiated by a trained community health worker or by women themselves. Groups can involve participatory cycles of action and learning ${ }^{1}[2]$.

- Group ANC/PNC typically consists of 8-12 women of similar gestational age, with 1-2 group leaders who employ a highly participatory, facilitative style while guiding women through group ANC/PNC sessions. The number of group sessions can be tailored to match global and local guidelines for required number of contacts. Each group session has a plan that includes clinical care and client education, as well as discussion throughout. Clinical care by a provider is provided during meetings, and women are engaged in self-assessments (e.g., blood pressure, weight, experiencing any danger signs). Meetings are counted as ANC visits/contacts. The same group of women and facilitators attend all sessions together to create a sense of stability, trust, and community within the group [3].

stories, and access resources and services related to $\mathrm{MNH}$ including for pre-eclampsia and eclampsia. The result is self-empowered women who have an increased awareness of maternal health issues, improved experiences of care, and ultimately, higher rates of maternal and child survival.

\section{WHY IS THE PRACTICE IMPORTANT?}

\section{Supports self-education around health and posi- tive health behaviors}

Participatory women's groups and group ANC/PNC are fora for women to learn about and discuss an array of

\footnotetext{
${ }^{1}$ Participatory learning and action cycles involve a four-phase process facilitated by a trained facilitator. The first phase is identifying and prioritizing problems during pregnancy, childbirth, and postpartum; the second phase is planning activities; the third phase is implementing strategies to address the problems discussed in phase one; and the fourth phase involves assessing the activities [1].
} 


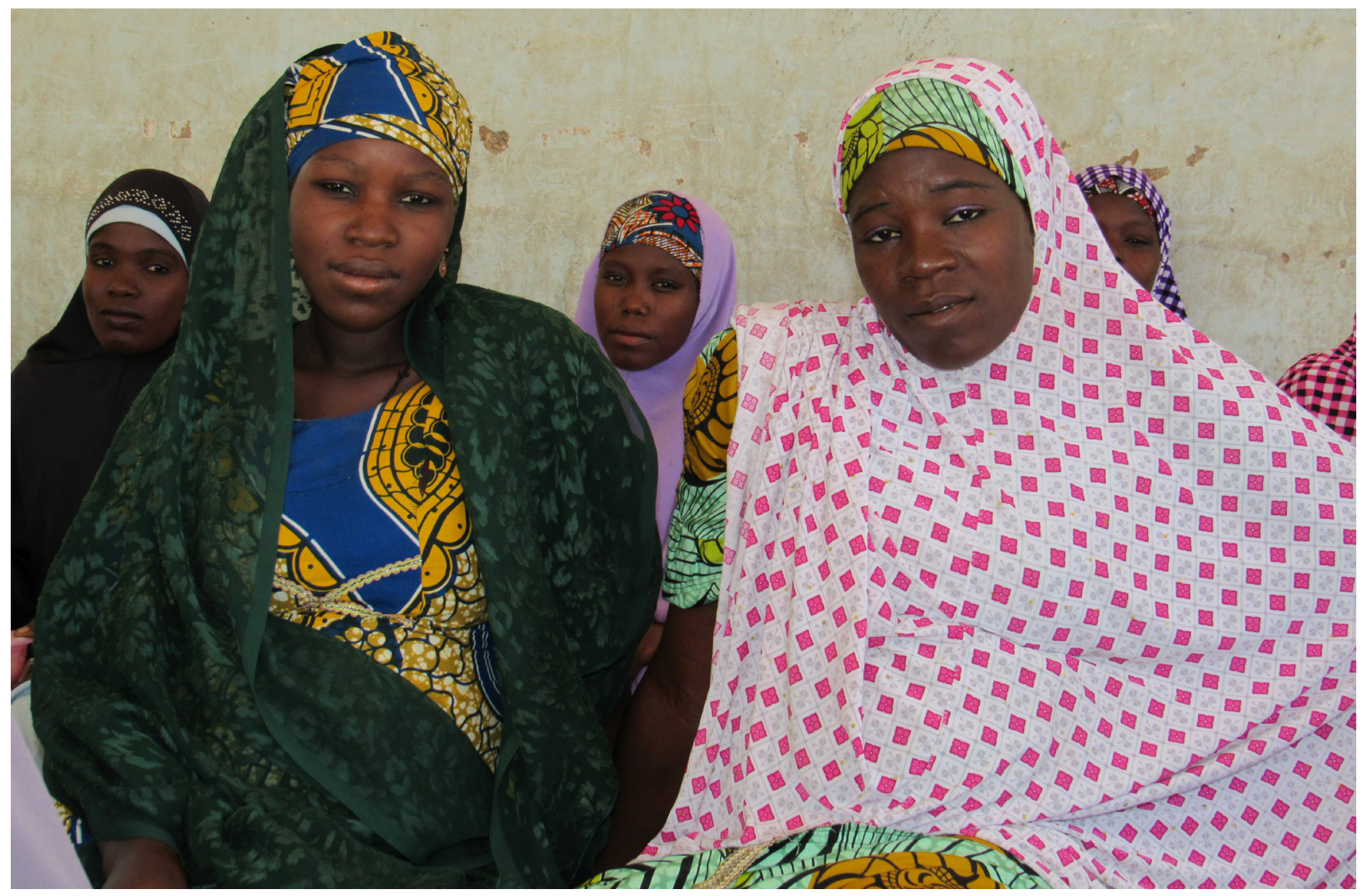

aspects of their health during and after pregnancy (e.g. nutrition, hypertension, etc.), as well as support one another in enacting positive health behaviors, including attending ANC and having a facility delivery.

\section{Encourages informed decisions regarding health- care for women and their families}

Participatory women's groups and group ANC/PNC not only offer vital information for the health of women, but also can support the empowerment of women to make decisions that are best for them regarding their healthcare.

\section{FOR PARTICIPATORY WOMEN'S GROUPS SPECIFICALLY...}

\section{Raise awareness on maternal and newborn health, including pre-eclampsia and eclampsia, in communities}

Women's groups can enable the vast spread of health information to women and their communities. For example, through the Ending Eclampsia project in Cross River state, Nigeria, women's groups leaders held 289 sessions and 16,031 contacts were made during the 9-month intervention period [9]. Among a sub-set of women in the intervention group, women's knowledge about maternal health complications (including having heard of preeclampsia and that pre-eclampsia is associated with high blood pressure and proteinuria, and were aware of at least three danger signs in pregnancy) improved from baseline to endline.

\section{FOR GROUP ANC SPECIFICALLY...}

\section{Builds social support among women through an improved care experience}

Participants of group ANC describe improved relationships with their peers and health care providers. Qualitative research in Rwanda found that one of the barriers to ANC was perceptions that providers would treat women poorly, but after group ANC, women reported experiencing respect and warmth from group ANC providers. Women also reported feeling supported by their peers [25]. Additionally, women who participated in the Ending Eclampsia women's groups intervention in Cross River State, Nigeria reported better ANC clinical experiences including higher quality of care [9]. 
Group ANC offers women higher quality of care compared to individual ANC

A study by Grenier et al found that women who participated in group ANC received higher quality of care compared to those receiving individual ANC. Quality of care was defined as receiving eight key interventions during pregnancy: being provided with a long-lasting insecticidal net during ANC, never running out of iron and folic acid supplements, HIV status known before delivery, syphilis test completed, receipt of three or more doses of intermittent preventive treatment in pregnancy for malaria, blood pressure recorded at each ANC visit, comprehensive counseling received, and danger signs assessed at all ANC visits [19].

\section{Links communities and community health workers with primary health care providers}

Through coordinated efforts, communities and community health workers can be effectively linked to primary health care providers for health care and referrals, respectively. For example, the Ending Eclampsia project in Sindh province, Pakistan successfully enhanced collaboration between community midwives (CMWs) and community health workers known as lady health workers (LHWs) by encouraging LHWs to refer pregnant women to group ANC conducted by community midwives [26].

\section{HOW TO DO IT: IMPLEMENTATION EXPERIENCE}

\section{Trained facilitators leading community women's groups in Nigeria [9]}

As part of the Ending Eclampsia project, the Population Council conducted implementation research in Cross River state in Nigeria to test the feasibility and utility of employing women's groups as community platforms to increase women's access to quality antenatal services using pre-eclampsia screening as the entry point.

The intervention included workshops for women's groups leaders on how to deliver relevant health information using pictorial job aids (flip charts) during participatory women's group meetings. Women's groups leaders also developed songs that depicted key messages such as the importance of early registration for ANC, adhering to provider advice and referrals to higher level health facilities, family planning and birth spacing, emergency maternal services, and the importance of facility delivery. This process was repeated in churches and other venues where women leaders routinely provide health talks.

Women in the intervention group had improved knowledge of maternal health complications, reported higher quality of care, were more likely to have their blood pressure checked and explained, and were more likely to ask providers questions and receive the results of their health checks. In intervention communities, ANC utilization, as well as the number of women who had their blood pressure and urine tested, increased.

\section{LHWs referring women to Group ANC/PNC led by Community Midwives in Pakistan [26]}

Implementation research conducted by the Population Council in Sindh Province, explored opportunities to enhance collaboration between CMWs and $\mathrm{LHWs}^{2}$ by encouraging LHWs to refer pregnant women for group ANC sessions conducted by local CMWs; and to improve ANC and PNC and counseling skills of CMWs, enabling them to manage postpartum hypertension.

CMWs were trained to conduct group sessions for ANC and PNC, and LHWs were sensitized/oriented to refer pregnant women to CMWs for group ANC and PNC. At endline, almost half of CMWs in the study reported that they arranged group ANC meetings in coordination with LHWs. Based on focus group discussions with women who attended Group ANC meetings with CMWs, women reported having a greater understanding of pre-eclampsia, severe pre-eclampsia, and eclampsia, and potentially fatal outcomes of persistent high blood presure during pregnancy, compared to women in areas where such meetings did not take place.

All LHWs who participated in the qualitative research expressed satisfaction with the group ANC sessions and observed that awareness among community women had increased after taking part in these sessions. LHWs themselves felt more confident in referring clients to CMWs. LHWs felt that group ANC is the best forum for community women to receive and exchange knowledge that can save the lives of pregnant women. Furthermore, when community women travel in groups to attend group ANC, it is cost-effective for them. Group ANC also proved to be a good platform for joint learning amongst community

\footnotetext{
2 Pakistan's MOH supports the CMW and LHW programs. CMWs are a cadre of rural midwives with a minimum of ten years of education. They participate in 18 months of training and are expected to establish private practices in their home villages. LHWs are affiliated with government health facilities and are trained, supervised, and given medical supplies to distribute as well as a small allowance of approximately $\$ 300$ per year [27].
} 
women where they can share their own pregnancyrelated problems with each other and obtain better advice.

\section{Facility-based group ANC in Kenya and Nigeria [19]}

Jhpiego conducted a two-country, two-phase cluster randomized controlled trial (cRCT) comparing group ANC to individual ANC in two African LMICs - Nigeria and Kenya. This group care model begins during pregnancy (five meetings); and continues for a year following birth for mothers and their babies (four meetings). The aim is to: 1) improve the quality of facility-based care (i.e., content, client satisfaction) and 2) increase retention in care and the use of key services. In both countries, women in the intervention arm were more likely to receive quality $\mathrm{ANC}^{3}$ and to attend at least four ANC visits.

In both countries, women in the intervention arm were more likely to complete all seven recommended BP/ CR components. ${ }^{4}$ In Nigeria women in the intervention arm were more likely to have a facility-based delivery compared to those in the control arm. In Nigeria, there was no significant difference in attendance at PNC, but median time from delivery to first facility-based PNC visit was two days earlier in the intervention versus the control $\operatorname{arm}(p=0.010)$. In Kenya, women in the intervention arm were significantly more likely to attend at least one PNC visit and attend their first PNC visit earlier-by a median difference of seven days. Group ANC was feasible and acceptable to women and providers. More than one year after study enrollment ceased, all intervention sites continued to provide Group ANC.

\section{Pregnancy Clubs in Kenya [28, 29]}

Management Sciences for Health (MSH), in partnership with the Kakamega County health management team and the Kenya Progressive Nurses Association (KPNA), implemented a group ANC model called Lea Mimba in Swahili, which translates to 'take care' in Swahili. Women met in groups, also called pregnancy clubs, with health providers seven times throughout their pregnancies. They shared their experiences, learned essential health information and self-care practices including checking blood pressure and weight, and supported one another emotionally and socially.
A mixed- methods study was conducted to assess the effect and acceptability of the group ANC model. The study found a higher proportion of women who made four or more ANC visits in the six intervention facilities compared to baseline data for these facilities from the national health management information system (HMIS) $(76.3 \%$ vs. $50 \%$, respectively). Note that the differences in these figures should be interpreted with caution as they were measured differently. Satisfaction with ANC care nearly doubled from baseline to endline, and this effect was greater among young women. There were no significant changes in knowledge or women's empowerment. Finally, although most women had blood pressure and weight recorded in their calendar books, the study team cautioned that some women may not have understood the meaning of their measurements.

\section{THESE APPROACHES WORK BEST WHERE...}

- Relevant stakeholders- including political and local leadership, facility staff implementers, and community-based organizations- are engaged and support these groups.

- The local context is considered, particularly the level and nature as to what is considered private or open for discussion in society.

- Trained providers of the appropriate cadre lead women's groups and group ANC/PNC and encourage active participation and discussion.

\section{WOMEN'S GROUPS WORK BEST WHERE...}

- Groups maintain fidelity to a participatory approach, particularly for participatory learning and action groups, and where communities take action based on what is discussed in the groups.

- Women are willing to and desire to participate and share their experiences.

\footnotetext{
${ }^{3}$ Quality of care was defined as the receipt of eight interventions during ANC: LLIN provided during ANC, Never ran out of IFAS tablets, HIV status known before delivery, Syphilis testing completed, IPTP 3+, Blood pressure recorded at every ANC visit, comprehensive counseling (e.g., comprehensive counseling included postpartum family planning options; lactational amenorrhea method; immediate breastfeeding; exclusive breastfeeding; danger signs during pregnancy; care-seeking for danger signs; eating extra food while pregnant and breastfeeding; newborn danger signs; prevention of sexually transmitted infections; use of LLIN; use of IFAS; birth planning); assessment of five danger signs at every visit included: asking woman about pain, fever, bleeding, leakage of fluids, and reduced or no fetal movement

${ }^{4}$ E.g., identified a facility; made a transportation plan; identified a companion; saved money; agreed on a decision-maker; agreed on an alternate decision maker; and prepared a birth kit to bring to the facility (items as recommended by each facility)
} 


\section{GROUP ANC WORKS BEST WHERE...}

- Groups are thoughtfully integrated into existing health systems. The nature of these linkages may vary across different sociocultural political contexts. For example, CHWs are introduced to those leading group ANC/PNC sessions (typically midwives) to encourage referrals.

- There are enough women of the same gestational age to make up a group for group ANC/PNC.

- There is a large private space so that women are comfortable participating and sharing, particularly for group ANC/PNC.

- Various time slots are available for group ANC as women may have different childcare or work responsibilities at different points of the day.

- There is trust between the community and all levels of providers including community health workers and midwives.

\section{CHALLENGES TO CONSIDER}

- Low population coverage of the intervention and a low proportion of newly pregnant women enrolled in women's groups could contribute to challenges in observing any population-level health impacts. In a CRCT in Bangladesh, there was not a greater reduction in neonatal mortality in intervention communities compared to control communities, as was seen in similar trials in Nepal and India, potentially due to low population coverage and a low proportion of newly pregnant women enrolled. [30] In a subsequent CRCT of participatory women's groups in Bangladesh with a higher population coverage of the intervention, reductions in neonatal mortality were observed [31].

- Results from a cRCT of women's group with action learning cycles in urban slums in Mumbai did not find differences at the population-level in healthcare or mortality [32]. The authors posited that contextual challenges including multiple sources of healthcare, inequitable access to services, and varying socioeconomic statuses could have contributed [32]. Interventions should target the most vulnerable families, links between communities and service providers should be enhanced, and quality of care improvements should be initiated in both public and private sectors.
- Lack of retention of women's groups facilitators, an increased workload of facilitators, and lack of refresher training and continuous support of facilitators are challenges to successful women's group implementation [30].

- Gender norms around women's decision-making and mobility may also affect successful implementation of women's groups. For example, in some contexts, women may face issues if they are required to ask their husbands or mothers-in-law for permission to join a women's group [30].

\section{PRIORITY RESEARCH QUESTIONS}

\section{Women's Groups}

- What are the essential elements (e.g. number of sessions, length of sessions, materials distributed, etc.) of effective women's groups meetings?

- What is the most effective balance of information dissemination, dialogue between women and the facilitator, and dialogue amongst women themselves?

- What are the strengths, challenges, and tradeoffs of group-based participatory learning models in promoting self-care from community and health systems perspectives?

- How do participatory women's groups work in urban areas compared to rural areas?+

- What are the effects of participatory women's groups in conjunction with quality improvements measures of health services and on care seeking behaviors?+

- What are the non-health benefits of participatory women's groups (e.g. are women empowered in other aspects of their lives than health)?+

- What are the potential harms (e.g. negative social capital such as restrictions to individual freedom and exclusion of outsiders) of participatory women's groups?+

*Adapted from the WHO guidelines on antenatal care [7].

Incorporates research recommendations from the GANC Collaborative [33].

+Incorporates research recommendations from the WHO recommendation on women's groups [1]. 
- How do we measure participatory women's groups' effect on the value of women by women themselves and by society at large?+

- How can women's groups synergize with ANC/PNC?

\section{Group ANC/PNC}

- What are the impacts of group ANC/PNC on the health system and service delivery (e.g. staffing needs and schedules, consequences of other facility services, cost, and training and supervision)?

- How does group ANC/PNC effect health service utilization (e.g. facility-based delivery, ANC and postnatal care attendance, and family planning uptake)?

- Is group ANC/PNC acceptable and feasible for women and healthcare providers?*

- What is the effect of group ANC/PNC on the uptake of healthy behaviors, increased health literacy and self-efficacy?

- What is the quality of care, including the provision and experience of care?

- What are the effects of group ANC/PNC on respectful care?

- Should women with complicated pregnancies be offered group ANC/PNC in addition to receiving specialized care?*

- What is the impact of group ANC/PNC for women who have little to no financial access or economic empowerment and are not empowered to make decisions for themselves?

- What is the impact of group ANC/PNC for unmarried pregnant women and girls, particularly in regards to building agency?

- What are the context-specific effects of group ANC/ PNC on maternal and perinatal health outcomes and coverage outcomes (ANC contacts and facility-based births)?*

\section{Additional research areas for both women's groups} and group ANC/PNC include:

- Cost-effectiveness of the interventions

- Policy implications, scalability, and sustainability
- Effective strategies for male engagement (for example, individual ANC encourages male partner participation and accompaniment - how can male engagement be incorporated into women's groups and group ANC)?

- Validated ways to measure key concepts including empowerment, experience of care, trust, social capital, self-efficacy, and agency

- Effective ways to address gender-related barriers to participation in women's groups and group ANC such as addressing potential tension with men in contexts where women have limited autonomy around their mobility * +

\section{TOOLS AND RESOURCES}

- Sharma et al collated the literature on group ANC and present a generic group care model that could be adapted in LMICs [3].

- Centering Healthcare Institute has a bibliography of their published work available [34].

- Group Care Global has resources for group care models globally.

- Ending Eclampsia's Nigeria women's groups job aid is available in the appendix of the endline report [9].

- White Ribbon Alliance Self-Care resources including Self-Care Communication Toolkit; Self-Care Model and Impact Infographic; Self-Care: FAQs, Toolkit for advocates, decision makers, health professionals, program implementers; and Self-Care related videos. [5].

- WHO Consolidated guideline on self-care interventions for health: sexual and reproductive health and rights [6].

- WHO recommendation on community mobilization through facilitated participatory learning and action cycles with women's groups for maternal and newborn health [1].

- WHO recommendations on antenatal care for a positive pregnancy experience [7]. 


\section{REFERENCES}

1. WHO. "WHO recommendation on community mobilization through facilitated participatory learning and action cycles with women's groups for maternal and newborn health." https://apps.who.int/iris/bitstream/ handle/10665/127939/9789241507271_eng.pdf?sequence $=1 \&$ ua $=1$ (accessed.

2. R. Preston et al., "How and why do participatory women's groups (PWGs) improve the quality of maternal and child health $(\mathrm{MCH})$ care? A systematic review protocol," BMJ Open, vol. 9, no. 9, p. e030461, Sep 5 2019, doi: 10.1136/ bmjopen-2019-030461.

3. J. Sharma, M. O'Connor, and R. Rima Jolivet, “Group antenatal care models in low- and middle-income countries: a systematic evidence synthesis," (in eng), Reproductive health, vol. 15, no. 1, p. 38, Mar 5 2018, doi: 10.1186/s12978-018-0476-9.

4. W. R. Alliance. "Self Care: A Cost Effective Solution for Maternal, Newborn, \& Child Health for All “ https://www. whiteribbonalliance.org/wp-content/uploads/2017/11/ White-Ribbon-Alliance-Self-care-Policy-Brief.pdf (accessed.

5. W. R. Alliance. "Resources: Self-care." https://www.whiteribbonalliance.org/self-care (accessed.

6. WHO, "WHO consolidated guideline on self-care interventions for health: sexual and reproductive health and rights," World Health Organization, Geneva, 2019.

7. WHO. "WHO recommendations on antenatal care for a positive pregnancy experience." https://apps.who.int/iris/bitstream/handle/10665/250796/9789241549912-eng. pdf?sequence $=1$ (accessed.

8. H. A. Harris-Fry et al., "Formative evaluation of a participatory women's group intervention to improve reproductive and women's health outcomes in rural Bangladesh: a controlled before and after study," (in eng), Journal of epidemiology and community health, vol. 70, no. 7, pp. 663-70, Jul 2016, doi: 10.1136/jech-2015-205855.

9. P. Sripad, E. Nwala, T. McClair, S. Ishaku, and C. Warren, “Engaging Community Women's Groups to Improve Maternal Health Care Delivery in Cross River: Implementation Research Report," Population Council, 2019.

10. N. Saggurti et al., "Effect of health intervention integration within women's self-help groups on collectivization and healthy practices around reproductive, maternal, neonatal and child health in rural India," (in eng), PLoS One, vol. 13 , no. 8 , p. e0202562, 2018, doi: 10.1371/journal. pone.0202562.

11. S. Sharma, E. Teijlingen, J. M. Belizan, V. Hundley, P. Simkhada, and E. Sicuri, "Measuring What Works: An Impact Evaluation of Women's Groups on Maternal Health Uptake in Rural Nepal," (in eng), PLoS One, vol. 11, no. 5, p. e0155144, 2016, doi: 10.1371/journal.pone.0155144.
12. L. Mangham-Jefferies, C. Pitt, S. Cousens, A. Mills, and J. Schellenberg, "Cost-effectiveness of strategies to improve the utilization and provision of maternal and newborn health care in low-income and lower-middle-income countries: a systematic review," (in eng), BMC pregnancy and childbirth, vol. 14, p. 243, Jul 22 2014, doi: 10.1186/14712393-14-243.

13. E. Kidney et al., "Systematic review of effect of community-level interventions to reduce maternal mortality," BMC pregnancy and childbirth, vol. 9, p. 2, Jan 20 2009, doi: 10.1186/1471-2393-9-2.

14. B. B. Sharma, L. Jones, D. J. Loxton, D. Booth, and R. Smith, "Systematic review of community participation interventions to improve maternal health outcomes in rural South Asia," BMC pregnancy and childbirth, vol. 18, no. 1, p. 327 , Aug 10 2018, doi: 10.1186/s12884-018-1964-1.

15. M. C. Jennings et al., "Comprehensive review of the evidence regarding the effectiveness of community-based primary health care in improving maternal, neonatal and child health: 2. maternal health findings," (in eng), Journal of global health, vol. 7, no. 1, p. 010902, Jun 2017, doi: 10.7189/jogh.07.010902.

16. A. Prost et al., “Women's groups practising participatory learning and action to improve maternal and newborn health in low-resource settings: a systematic review and meta-analysis," Lancet, vol. 381, no. 9879, pp. 1736-46, May 18 2013, doi: 10.1016/S0140-6736(13)60685-6.

17. P. Tripathy et al., "Effect of participatory women's groups facilitated by Accredited Social Health Activists on birth outcomes in rural eastern India: a cluster-randomised controlled trial," (in eng), The Lancet. Global health, vol. 4, no. 2, pp. e119-28, Feb 2016, doi: 10.1016/s2214$109 \times(15) 00287-9$.

18. N. Seward et al., "Effects of women's groups practising participatory learning and action on preventive and care-seeking behaviours to reduce neonatal mortality: A meta-analysis of cluster-randomised trials," (in eng), PLoS Med, vol. 14, no. 12, p. e1002467, Dec 2017, doi: 10.1371/journal. pmed.1002467.

19. L. Grenier et al., "Impact of group antenatal care (G-ANC) versus individual antenatal care (ANC) on quality of care, ANC attendance and facility-based delivery: A pragmatic cluster-randomized controlled trial in Kenya and Nigeria," PLoS One, vol. 14, no. 10, p. e0222177, 2019, doi: 10.1371/journal.pone.0222177.

20. J. R. Lori, M. Chuey, M. L. Munro-Kramer, H. Ofosu-Darkwah, and R. M. K. Adanu, "Increasing postpartum family planning uptake through group antenatal care: a longitudinal prospective cohort design," (in eng), Reproductive health, vol. 15, no. 1, p. 208, Dec 17 2018, doi: 10.1186/s12978018-0644-y. 
21. F. Jafari, H. Eftekhar, A. Fotouhi, K. Mohammad, and S. Hantoushzadeh, "Comparison of maternal and neonatal outcomes of group versus individual prenatal care: a new experience in Iran," Health Care Women Int, vol. 31, no. 7, pp. 571-84, Jul 2010, doi: 10.1080/07399331003646323.

22. C. L. Patil et al., "Randomized controlled pilot of a group antenatal care model and the sociodemographic factors associated with pregnancy-related empowerment in sub-Saharan Africa," (in eng), BMC pregnancy and childbirth, vol. 17, no. Suppl 2, p. 336, Nov 8 2017, doi: 10.1186/ s12884-017-1493-3.

23. R. R. Jolivet, B. V. Uttekar, M. O'Connor, K. Lakhwani, J. Sharma, and M. N. Wegner, "Exploring perceptions of group antenatal Care in Urban India: results of a feasibility study," (in eng), Reproductive health, vol. 15, no. 1, p. 57, Apr 32018 , doi: 10.1186/s12978-018-0498-3.

24. T. Lundeen et al., 'Nurses' and midwives' experiences of providing group antenatal and postnatal care at 18 health centers in Rwanda: A mixed methods study," PLoS One, vol. 14, no. 7, p. e0219471, 2019, doi: 10.1371/journal. pone.0219471.

25. A. Musabyimana et al., "Before and after implementation of group antenatal care in Rwanda: a qualitative study of women's experiences," Reproductive health, vol. 16, no. 1, p. 90, Jun 27 2019, doi: 10.1186/s12978-019-0750-5.

26. Population Council, "Expanding Services to Detect, Manage, and Prevent Pre-eclampsia/Eclampsia in District Tando Allahyar in Sindh Province of Pakistan," Population Council, 2019.

27. Z. Mumtaz, S. Salway, C. Nykiforuk, A. Bhatti, A. Ataullahjan, and B. Ayyalasomayajula, "The role of social geography on Lady Health Workers' mobility and effectiveness in Pakistan," Soc Sci Med, vol. 91, pp. 48-57, Aug 2013, doi: 10.1016/j.socscimed.2013.05.007.

28. M. Kenya, "Lea Mimba: A Group Based Model for Antenatal Care in Kakamega County, Kenya," 2019. [Online]. Available: https://www.msh.org/resources/leamimba-techical-brief
29. M. Kenya, “Lea Mimba (Take Care of Your Pregnancy) Pregnancy Clubs: A Group Based Model for Antenatal Care in Kakamega County, Kenya," 2019. [Online]. Available: https:// www.msh.org/resources/lea-mimba-evidence-brief

30. K. Azad et al., "Effect of scaling up women's groups on birth outcomes in three rural districts in Bangladesh: a cluster-randomised controlled trial," Lancet, vol. 375, no. 9721, pp. 1193-202, Apr 3 2010, doi: 10.1016/S01406736(10)60142-0.

31. E. Fottrell et al., "The effect of increased coverage of participatory women's groups on neonatal mortality in Bangladesh: A cluster randomized trial," JAMA Pediatr, vol. 167, no. 9 , pp. 816-25, Sep 2013, doi: 10.1001/jamapediatrics.2013.2534.

32. N. S. More et al., "Community mobilization in Mumbai slums to improve perinatal care and outcomes: a cluster randomized controlled trial," PLoS Med, vol. 9, no. 7, p. e1001257, 2012, doi: 10.1371/journal.pmed.1001257.

33. G. Collaborative. "Group Antenatal Care." https:// www.ganccollaborative.com/group-antenatal-care-1 (accessed.

34. C. H. Institute. "Advancing maternal child health through evidence-based practice." https://www.centeringhealthcare. org/why-centering/research-and-resources (accessed.

Acknowledgments: This document was originally drafted by Tracy McClair. Critical review and helpful comments were provided by Emily Hillman, Sharif Hossain, Karen Kirk, Kathryn Mimno, Ali Mohammad Mir, Yordanos B Molla, Mary Ndu, Pooja Sripad, Suzanne Stalls, Marianne Vidler, and Charlotte Warren.

For further information please contact us at MNH-PCDC@popcouncil.org

Suggested citation: High-Impact Practices (HIPs) in Maternal and Newborn Health. Encouraging empowerment and self-care through participatory women's groups and group antenatal care: supporting women to be at the center of their own care as active participants. Washington (DC): Population Council; 2020. Available from: https://www.popcouncil.org/research/ending-eclampsia1
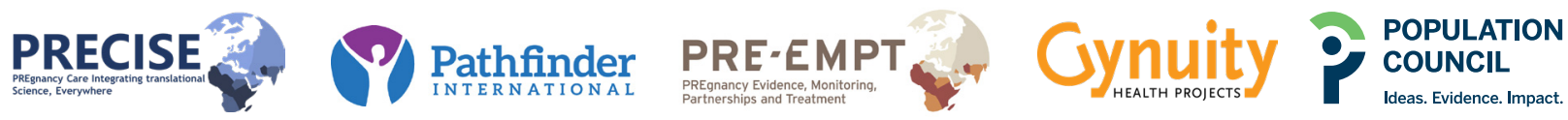